\title{
Mesoscopic Solvent Dynamics in a Real Dimensional System
}

\author{
Song Hi Lee \\ Department of Chemistry, Kuthgstng Lniversitv, Busan 608-736, Korea \\ Received October 5, 2004
}

\begin{abstract}
Hỵdrodynamic simulations of mesoscopic solvent have been performed by multi-particle collision algorithm in a real dimensional system without and with the random shifting of the grid. A systematic conversion of the dimensionless units to a real dimensional syrstem was confirmed by jump rates of solvent particles. Speed distributions of solvent particles obtained from the simulations agreed very well with the Maxwell-Boltzmann distributions. Solvent viscosities obtained from the simulations and from the conversion of units are exactly the same which confirmed the correct conversion of the units once again. The calculation of the friction coefficient of a massive Brownian particle in a mesoscopic solvent as a function of Brownian particle diameter was examined as an example.
\end{abstract}

Key Words : Mesoscopic solvent. Multi-particle collision. Real dimension

\section{Introduction}

Performing hydrodynamic simulations of complex fluids such as amphiphilic mixtures and polymer melts is a challenging task. For these systems. mesoscopic simulation methods are often more efficient and stable than conventional computational fluid dynanics algorithms and they have shown great promise for simulating flow in complicated geometries. Examples include lattice gas automata (LGA), ${ }^{1}$ lattice Boltzmann (LB) algorithmı. ${ }^{2}$ particle-based mesoscopic simulation techniques such as dissipative particle dynamics (DPD). ${ }^{3}$

In the LGA method. the fluid is modeled as a collection of particles which move along the links of a regular lattice and interact according to well-defined collision rules. If these collision rules preserve mass and momentum conservation, the correct hydrodynamic behavior can be obtained at macroscopic length scales. In the LB method, the time development of particle distribution functions with a set of fixed lattice velocities on a regular lattice is described by a discretized LB equation. Both of these lattice methods are very efficient due to the simplified collision dynamics and strong reduction of velocity space. However. this reduction is also their major drawback: they are not Galilean-invariant. a problem which restricts their use to conditions in which the flow velocity is small compared to the maximum lattice velocity. In addition, the LB approach is subject to a number of numerical instabilities. While off-lattice methods such as DPD do not suffer from these drawbacks. they are often complex and difficult to analyze analytically.

Recently Malevanets and Kapral described one such mesoscopic approach which we shall term multi-particle collision dynamics. ${ }^{45}$ The fluid is modeled by particles whose positions and velocities are continuous variables. The system is coarse-grained into the cells of a regular lattice and there is no restriction on the number of particles in a cell. The evolution of the system consists of two steps: streaming

*e-mail: shlee ôstar.ks.ac.kr and collision. In the streaming step, the coordinate of each particle is incremented by its displacement during the time step. Collisions are modeled by a stochastic rotation of the relative velocities of every particle in each cell. The dynamics is explicitly constructed to conserve mass, momentum, and energy, and the collision process is the simplest consistent with these conservation laws. It has been shown that there is an $H$-theorem for the dynamics and that this procedure yields the correct hydrodynamic equations for an ideal gas. The mesoscopic multi-particle collision model has been used previously to study hydrodynamic flows: ${ }^{6 \cdot 9}$ cluster dynamics ${ }^{15}$ small $n$-alkane dynamics. ${ }^{11}$ polymer dynamics. ${ }^{12}$ phase segregation and colloidal suspensions. ${ }^{13^{3}}$ complex fluids, ${ }^{1+16}$ diffusion-influenced reaction dynamics. ${ }^{17}$ friction and diffusion of a Brownian particle, ${ }^{18}$ and friction between two Bromnian particles. ${ }^{19}$

However, all the simulation parameters are in the dimensionless units. To understand as fully as possible how multiparticle collision dynamics can be used to simulate hydrodynamic behavior it is necessary to convert the dimensionless units to a real dimensional system. Therefore this paper presents a systematic conversion of the simulation parameters and system properties in a real dimensional system.

The outline of the paper is as follows. The mesoscopic model of multi-particle collision following Malevanets and Kapral $^{4.5}$ and the random shifting of the grid ${ }^{5}$ are summarized in Section II. We then describe the systematic conversion of the simulation parameters and system properties from the dimensionless units to a real dimensional system in Section III. The results of the simulations for speed distribution, solvent viscosity. and friction of a massive Brownian particle are described in Section IV and the conclusions of the paper are given in Section V.

\section{Multi-particle Collision Algorithm and the Random Shifting of the Grid}

Consider a set of $N$ point-particles of mass $m$ which move 
in continuous space with a continuous distribution of velocities but discretely in time. The algorithm proceeds in two steps. In the first of these. a free streaming step. the positions of the solvent particles at time $t . r_{j}(t)$. are updated simultaneously according to

$$
\mathbf{r}_{\mathrm{i}}(\mathrm{t}+t)=\mathbf{r}_{\mathrm{i}}(\mathrm{t})+\mathbf{v}_{\mathrm{i}}(\mathrm{t}) t
$$

where $v_{i}(t)$ is the velocity of a particle and $\tau$ is the value of the discrete time step of the solvent. The second part of the algorithm is the collision step. The system of cubic with size $L$ is coarse-grained into $(L / \alpha)^{d}$ cells of a $d$-dimensional lattice with side $a$. There is no restriction on the total number of solvent particles in each cell (although the total number of particles in the system is conserved). Stochastic multiparticle collisions are performed within each individual cell. by rotating the velocity of each particle relative to the centerof-mass velocity $v_{\mathrm{cor}}(\mathrm{t})$ of all the particles within that cell.

$$
v_{i}(t)=v_{c m}(t)+R\left[v_{i}(t)-v_{c m}(t)\right] .
$$

$\mathbf{R}$ is a rotation matrix which rotates velocities by a fixed angle $\alpha$ around an axis generated randomly for each cell and at each time step.

The aim of the collision step is to transfer momentum between the particles while conserving the total momentum energy of each cell. Because mass. momentum. and energy are conserved locally the thermohydrodynamic equation of motion are captured in the continumum limit. Hence hydrodynamic interactions can be propagated by the solvent. Note. however. that any molecular details of the solvent are excluded: this allows the hydrodynamic interactions to be modeled with minimal computational expense

For small $\Lambda(=\lambda \alpha$, with the mean free path $\lambda)$. large numbers of particles in a given cell remains correlated over several time steps. These correlations are changed by the presence of flow, and Galilean-invariance is broken. One way of removing this dependence on the macroscopic velocity is to perform a random shift of the grid before performing the stochastic rotation: All particles are shifted by the same random vector with components in the interval $[-a / 2, a / 2]$ before the collision step. Particles are then shifted back by the same amount after the collision. This shifting procedure. in conjunction with the stochastic collision. leads to an additional contribution to the viscosity. In the streaming step. momentum is transferred directly from one cell to another when particles cross cell boundaries. This leads to a kinetic contribution to the viscosity. $\eta_{\text {kin. }}$ calculated previously ${ }^{4.5}$ However as mentioned above. there is also a rotational contribution. $\eta_{\text {rot }}$. The total kinematic viscosity therefore consists of the sum of three contributions. $\eta=\eta_{\text {kin }}+\eta_{\text {ric }}+\eta_{\text {mix. }}$ where $\eta_{\text {mix }}$ is a cross term resulting from both streaming and rotation. These additional contributions are zero in the original method. but when the random shifting procedure is applied, the rotational contribution is non zero: for $A \ll 1$. $\eta_{\text {rot }}$ is the dominant contribution to the viscosity.

\section{Conversion of Dimensionless Units to a Real Dimensional System}

In the dimensionless system. ${ }^{5} m=1, a=1$ and the number of cells was $L^{3}=32^{3}$ in 3 -dimension. The initial solvent distribution was generated by assigning positions randomly within the system with an average number of particles per unit cell $\rho=10$. Thus total number of particles was $N=$ 327680. The velocities were assigned from a uniform distribution $\left(-v_{\text {max }} \leq v_{\beta} \leq v_{\text {max. }} \beta=\mathrm{x}, \mathrm{y}, \mathrm{z}\right)$. where $\mathrm{v}_{\mathrm{max}}=$ $\left(3 k_{B} T / m\right)^{1: 2}$. The distribution relaxed rapidly $(t \sim 1000$ time steps) to the equilibrium Maxwell-Boltzmann form corresponding to the temperature $T$. Further $k_{B} T=1 / 3$ and $\tau=1$ were chosen. In summary. $m^{*}=1, a^{*}=1, \rho^{*}=10,\left(k_{B} T\right)^{*}=1 / 3$, and $t=1$ where the superscript ${ }^{*}$ indicates the dimensionless units.

For a real dimensional system. first. the mass of mesoscopic solvent particle is chosen as $m=3.995 \mathrm{~g} / \mathrm{mol}$ which is $1 / 10$ of that of Argon atom. The length of unit cell is chosen as $a=0.17 \mathrm{~nm}$ and the number density of the system is $\rho=$ $N / V=N / L^{3}=327680 /(32 \times 0.17 \mathrm{~nm})^{3}=2035.4 \mathrm{~nm}^{-3}$. Finally the temperature is chosen as $T=40.33 \mathrm{~K}$. With these fundamental parameters ( $m, a$, and $T$ ), the value of the discretized time step of the solvent, $\tau$ is determined by $\left(k_{B} T\right)^{*}=\left[\left(k_{B} T /\left(m a^{2} / \tau^{2}\right)\right]=1 / 3\right.$. Hence.

$$
\tau=\left(\frac{m a^{2}}{3 k_{B} T}\right)^{1 / 2}=\frac{2.15145}{T^{1: 2}}(\mathrm{ps})
$$

For $T=40.33 \mathrm{~K} . \tau=0.33878 \mathrm{ps}$.

In order to confirm that the above conversion is correct. both simulations of dimensionless and dimensional sy stems with the above simulation parameters were performed and jump rates of particles were compared for both systems. Jump rate is defined by the number of moving particles within a time step. $\tau$. For example, after a time step. if $N_{1}$ particles move to the neighboring cell. $N_{2}$ particles cross two neighboring cells and so on. jump rate is equal to $N_{1}+2 N_{2}+$ $3 N_{3}+\cdots$. Table 1 shows the result for the jump rate of both șistems without the random shifting of the grid. The snall

Table 1. Jump rates for dimensionless and dimensional systems without the random shifting of the grid. Each run is averaged for logotime steps. Dimensional I, 2 and 3 represent the systems at $\mathrm{T}=40.33,48.4$ and $85.0 \mathrm{~K}$, respectively.

\begin{tabular}{cccccc}
\hline System & Run 1 & Run 2 & Run 3 & Run 4 & Average \\
\hline Dimensionless & 150952 & 150943 & 150945 & 150951 & $150948 \pm 4$ \\
Dimensional 1 & 150959 & 150957 & 150958 & 150959 & $150958 \pm 1$ \\
Dimensional 2 & 150947 & 150945 & 150950 & 150941 & $150946 \pm 3$ \\
Dimensional 3 & 150950 & 150954 & 150955 & 150947 & $150952 \pm 3$ \\
\hline
\end{tabular}




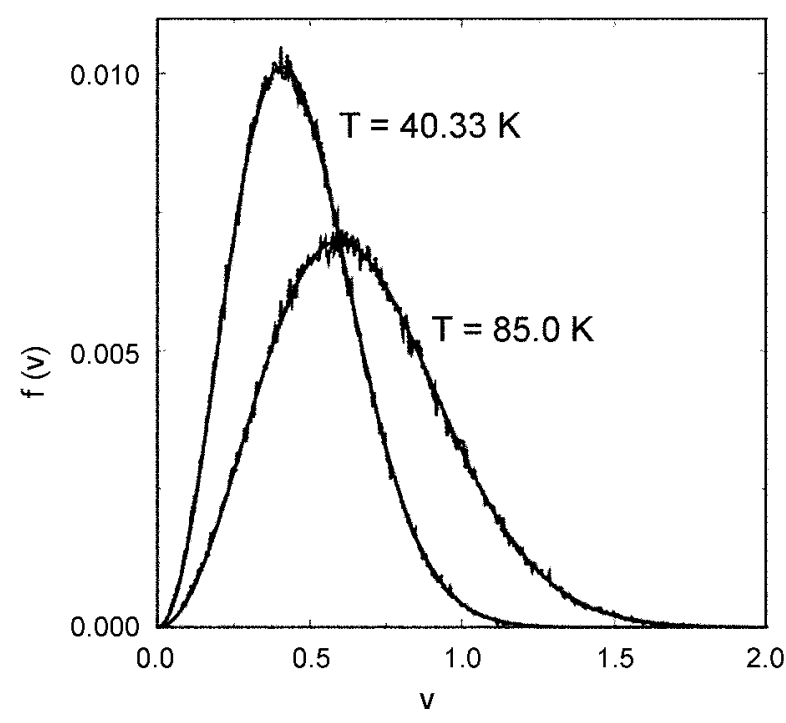

Figure 1. Comparison of speed distribution: Maswell-Boltzmann speed distributions (solid line); histograms of $\mathrm{v}$ distributions computed in the simulations (dotted line). The histograms are obtained from only one configuration of mesoscopic solvent particles.

deviation is within statistical error and we could confirm that Eq. (3) is correct. Further confirmation for the conversion of units will be discussed in solvent viscosity calculation in the below.

\section{Speed Distribution, Viscosity Calculation, and Friction of a Brownian Particle}

We have carried out multi-particle collision simulations for the systems of mesoscopic solvent in a real dimension at $T=40.33 .48 .4$, and $85.0 \mathrm{~K}$. The corresponding values of $\tau$ are $0.33878,0.30925$, and $0.23336 \mathrm{ps}$ from Eq. (3). respectively: The equilibrium states were achieved within 1000 time steps. Table 1 contains the jump rates of solvent particles at $T=48.4$ and $85.0 \mathrm{~K}$ without the random shifting of the grid.

Figure 1 compares speed distributions of mesoscopic solvent particles from the results of the simulations and from the Maxwell-Boltzmann distribution at $T=40.33$ and 85.0 $\mathrm{K}$. The two speed distributions agree very well even though the results from the simulations were obtained from just one configuration of mesoscopic solvent particles of $N=$ 327680 .

Solvent viscosity coefficient is obtained by

$$
\eta=\lim _{\mathrm{T} \rightarrow \infty} \frac{m^{2} \rho}{2 k_{B} T N T \tau} \sum_{t, t<\mathrm{T}} \sum_{i, j} v_{x j}(t) \Delta \xi_{y, j} v_{x y}\left(t^{\prime}\right) \Delta \xi_{y j}\left(t^{\prime}\right)
$$

where $\xi$ is the coordinate of a coarse-grained cell of the system. In the dimensionless units the dimensionless viscosity coefficient is $\eta^{*}=1.97$ obtained from the stress autocorrelation function." To convert the dimensionless viscosity to the real viscosity we have $\eta=(\mathrm{m} / \mathrm{\alpha}) \eta^{*}$ with $m=$ $3.995 \mathrm{~g} / \mathrm{mol}$. $\tau=0.33878 \mathrm{ps}$ and the cell length $a=0.17 \mathrm{~nm}$.

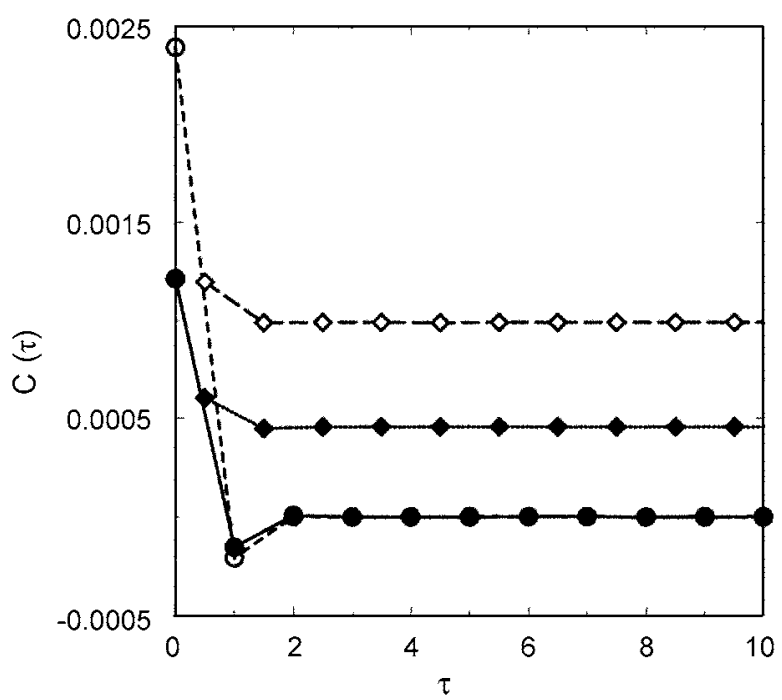

Figure 2. The stress auto-correlation function, $\mathrm{C}(t)$, and its running integral without (black symbols) and with the random shifting of the grid (open symbols). The circles show the computed stress auto-conelation function and the diamonds represent the values of the partial summation of the stress auto-correlation function.

Thus. $\eta=2.269 \times 10^{-4} \mathrm{~kg} / \mathrm{m} \mathrm{s}$. From the simulation for a real dimensional șistem of $T=40.33 \mathrm{~K}$ and $\tau=0.33878 \mathrm{ps}$. the second part of Eq. (4) is obtained. In Figure 2 we present the results of numerical simulations of the stress-stress autocorrelation function. $\mathrm{C}(\boldsymbol{t})$. and its ruming integral without the random shifting of the grid. The obtained running integral divided by $2 N \mathrm{~T}$ is equal to $4.778 \times 10^{-4} \mathrm{rm}^{-1} / \mathrm{ps}^{2}$. The first part of Eq. (4), $m^{2} \rho / k_{B} T \tau$ with $m=3.995 \mathrm{~g} / \mathrm{mol} . \rho=$ $2035.4 \mathrm{~nm}^{-3} . T=40.33 \mathrm{~K}$. and $\tau=0.33878$ ps gives $286.0 \mathrm{~kg}$ ps $/$ mol nm ${ }^{5}$. Combining these two parts. we obtain $\eta=$ $0.13665 \mathrm{~kg} / \mathrm{mol}$ ps $1 \mathrm{~m}=2.269 \times 10^{-4} \mathrm{~kg} / \mathrm{m} \mathrm{s}$ without the random shifting of the grid. which is exactly the same as the value of the viscosity coefficient converted from the dimensionless viscosity coefficient $\eta^{*}=1.97$. Therefore the conversion of units was confirmed once again. Further the values of solvent viscosities at $T=48.4$ and $85.0 \mathrm{~K}$ are obtained as $\eta=2.486 \times 10^{-4}$ and $3.294 \times 10^{-4} \mathrm{~kg} / \mathrm{m} \mathrm{s}$ from both the conversion of unites and the results of the simulations at $T=48.4$ and $85.0 \mathrm{~K}$ without the random shifting of the grid. respectively.

In a previous study ${ }^{10}$ the viscosity coefficients of a mesoscopic solvent at $T=40.33 .48 .4$ and $85.0 \mathrm{~K}$ were estimated as $\eta=5.0 \times 10^{-4} .65 \times 10^{-4}$ and $10.0 \times 10^{-4} \mathrm{~kg} / \mathrm{m}$ s. respectively. which are apparently inconsistent with the above values of at the same temperatures. The main reason for this disagreement is due to the choice of the discrete time step as $\tau=0.1 \mathrm{ps}$ in the previous study ${ }^{10}$ since all the other simulation parameters were the same for the mesoscopic solvent. The value of $\tau$ was not determined systematically which resulted in obtaining different $\eta$ with different $\tau$. The choice of $\tau$ only according to $\mathrm{Eq}$. (3) gives a correct dyrnamics of the mesoscopic solvent.

When the random shifting procedure is applied. the shear viscosity can be obtained in three dimensions ${ }^{i}$. 


$$
\eta=\frac{\tau}{N k_{B} T} \sum_{t=0}^{\infty}\left\langle\mathrm{I}_{2}(\hat{z} .0) \mid \mathrm{I}_{2}(\hat{z}, t)\right\rangle
$$

where the prime on the sum indicates that $t=0$ term has the relative weight $1 / 2$. There are two contributions to the reduced fluxes namely kinetic and rotational. so that

$$
\mathrm{I}_{2}(\hat{z}, t)=\mathrm{I}_{2}^{\mathrm{kjn}}(\hat{z} \cdot t)+\mathrm{I}_{2}^{\mathrm{T} T t}(\hat{z} \cdot t)
$$

where

$$
\mathrm{I}_{z}^{\mathrm{knn}}(\hat{z} . t)=-\frac{1}{\tau} \sum_{i} v_{i x}(t) \Delta \xi_{i}(t)
$$

and

$$
\mathrm{I}_{2}^{\mathrm{Tnt}}(\hat{z}, t)=-\frac{1}{\tau} \sum_{z} \Delta v_{i x}(t) \Delta \xi_{i z}^{\dagger}(t)
$$

Here. $\Delta \xi_{i}(t)=\xi_{i}(t+t)-\xi_{i}(t)$ and $\Delta \xi_{i}(t)=\xi_{i}(t+t)-\xi_{i}^{s}(t+$ $t)$. where $\xi(t+t)$ is the cell coordinate of particle $i$ at time $t$ $+t$ and $\xi_{i}^{s}(t+t)$ is the corresponding shifted particle cell coordinate. Contributions to $\mathrm{I}_{2}^{\text {kin }}$ come from the streaning step, whereas the collisions and shifts contribute to $\mathrm{I}_{2}{ }^{\text {rot }}$. There are corresponding kinetic, rotational, and mixed contributions to the shear viscosity. In our real dimensional system, performing the same MD simulation with the random shifting of the grid. the obtained viscosity is $\eta=$ $4.704 \times 10^{-4} \mathrm{~kg} / \mathrm{m} \mathrm{s}$ at $T=40.33 \mathrm{~K}$ with $t=0.33878 \mathrm{ps}$. Figure 2 shows the results of numerical sintulations of the stress-stress auto-correlation function, $\mathrm{C}(t)$. and its runing integral with the random shifting of the grid. In this case. $\lambda=$ $0.17 \mathrm{~lm}$ and $\Lambda=1$. The kinetic, rotational, and mixed contributions to the shear viscosity are $2.269 \times 10^{-4} .-0.292$ $\times 10^{-4}$, and $2.727 \times 10^{-4} \mathrm{~kg} / \mathrm{m} \mathrm{s}$, respectively.

As an exaniple for the use of the real dimensional system. the friction coefficients of a massive Brownian particle inumersed in a mesoscopic solvent have been calculated as a function of Brownian particle diameter at $T=40.33 \mathrm{~K}$. without and with the random shifting of the grid. The Brownian particle. fixed in the center of the simulation box. interacts with the mesoscopic solvent particles via the repulsive Lennard-Jones potential ${ }^{3 i}$ with $\varepsilon=1.00604 \mathrm{~kJ} / \mathrm{mol}$ and $\sigma=0.1 .0 .3 .0 .5,0.7 .0 .9$, and $1.0 \mathrm{~nm}$. The system evolves by Nenton's equations of motion which were integrated using the velocity Verlet algorithm. ${ }^{21}$ with a time step of $d t=750=0.0067756 \mathrm{ps}$. The simulations were carried out in a cubic box of volume $V=L^{3}$ with periodic boundary conditions. If the volume of the Brownian particle is $V_{B}=4 \pi \sigma^{3} / 3, V_{0}$ is defuned as the volume of the system occupied by solvent molecules, $V_{0}=V-V_{\mathrm{B}}$. The values of $L$ and $N(=327680)$ were chosen to fix the number density of solvent particles at $\rho=2035.4 \mathrm{~nm}^{-\hat{3}}$.

The friction coefficient of a Brownian particle can be calculated from the time integration of the force autocorrelation function:

$$
\zeta_{t}(t)=\frac{1}{3 k_{B} T} \int_{0}^{t} d \tau\langle\mathbf{F}(\tau) \cdot \mathbf{F}(0)\rangle
$$

where $\mathbf{F}$ is the force exerted on the Brownian particle and the momentum auto-correlation functions:

$$
C_{F}(t)=\frac{\langle\mathbf{P}(t) \cdot \mathbf{P}(0)\rangle}{\left\langle\mathrm{P}(0)^{2}\right\rangle}
$$

where $\mathbf{P}$ is the momentum of the Brownian particle or minus the total momentum of solvent particles since the total momentum of the system conserves. Eqs. (9) and (10) give three friction coefficients: $\zeta_{j}(t)=\zeta_{1} e^{-\zeta_{1} t * m}$ and $C_{p}(t)=$ $e^{-\zeta_{3} t: k m}$. It is found from our simulations that the decay rate of time integration of the force auto-correlation function ( $\left.\zeta_{y}\right)$ $\mathrm{Nm}$ ) is difficult to estimate due to its nonlinear slope and. on the other hand. the momentum auto-correlation function shows a regular exponential behavior which gives a welldefined friction coefficient $\left(\zeta_{3} / \mathrm{Nm}\right)$. It is also found that $\zeta_{1}$ and $\zeta_{3}$ agree very well. It is most interesting that for long times the values of $\zeta(t)$ in Eq. (9) can be obtained by the time derivative of $C_{p}(t)$ in Eq. (10):

$$
\begin{aligned}
\zeta_{f}(t) & =-N m \frac{d}{d t} C_{F}(t)=\frac{1}{3 k_{B} T}\langle\mathbf{F}(t) \cdot \mathbf{P}(0)\rangle \\
& =\frac{1}{3 k_{B} T} \lim _{s \rightarrow \infty} \frac{1}{s} \int_{0}^{s} d u \mathbf{F}(u+t) \cdot \mathbf{P}(u) \\
& =-\frac{1}{3 k_{B} T} \lim _{s \rightarrow \infty} \frac{1}{s} \int_{0}^{s} d u \mathbf{F}(u) \cdot \mathbf{P}(u+t) \\
& =\frac{1}{3 k_{B} T} \lim _{s \rightarrow \infty} \frac{1}{s} \int_{0}^{s} d u \int_{a}^{t} d t \mathbf{F}(u) \cdot \mathbf{F}(u+\tau) \\
& =\frac{1}{3 k_{B} T} \int_{i j}^{T} d \tau\langle\mathbf{F}(\tau) \cdot \mathbf{F}(0)\rangle
\end{aligned}
$$

where $\left\langle\mathrm{P}(0) \hat{>}>=3 k_{B} T N m\right.$ and $\mathbf{P}(t)=-\int_{0}^{t} d \tau \mathbf{F}(\tau)$ were used, and wice versa:

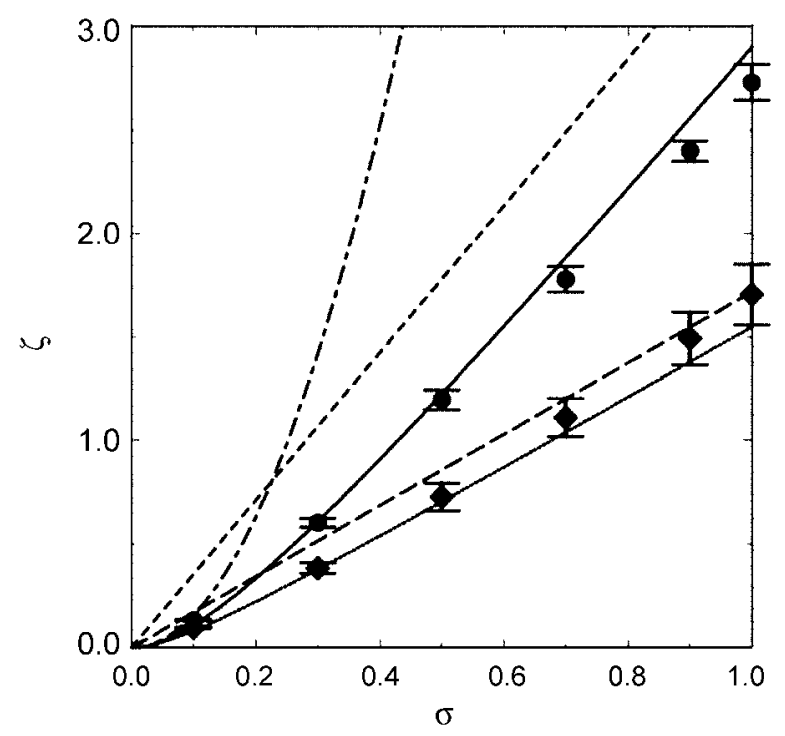

Figure 3. Friction coefficients as function of $\sigma$. Circles, 5 ; solid line, $\zeta_{i}$ and dashed line, $\xi_{s}$ with the random shifting of the grid. diamonds, $\zeta_{i}$, dotted line, $\zeta_{i}$ and long dashed line, $\zeta_{i}$ without the random shifting of the grid, and dot-dashed line, $\zeta_{m}$. 


$$
C_{P}(t)=1-\frac{1}{N m} \int_{0}^{t} d \tau \zeta_{f}(t) .
$$

In Figure 3 we show the friction coefficient as function of $\sigma$. The hydrodynamic contribution of the friction is given by $\zeta_{h}=4 \pi \eta \sigma$ with slip boundary condition and a rough estimate of the microscopic contribution to the friction can be obtained using a hard sphere binary collision model with the collision diameter chosen to be $\sigma \cdot \zeta_{\pi}=8 \rho \hat{\sigma}\left(2 \pi m k_{B} T\right)^{1 / 2} / 3$. An estimate of the friction that accounts for both microscopic and hydrodynamic contributions to the friction is given by $\zeta_{t}^{-1}=\zeta_{m}^{-1}+\zeta_{13}^{-1}+2$ In the case without the random shifting of the grid $\left(\eta=2.269 \times 10^{-4} \mathrm{~kg} / \mathrm{m} \mathrm{s}\right)$ the theoretical prediction $\left(\zeta_{t}\right)$ underestimates the simulation result $\left(\zeta_{1}\right)$, but in the case with the random shifting of the grid $\left(\eta=4.704 \times 10^{-4} \mathrm{~kg} / \mathrm{m} \mathrm{s}\right)$ the opposite is observed. When $\sigma$ is small the microscopic contributions $\left(\zeta_{\pi}\right)$ dominate the friction coefficient as expected, and as $\sigma$ increase. the hydrodynamic estunate of the friction lies close to the simulation value $\left(\zeta_{1}\right)$. For the largest values of $\sigma$. the hydrodynamic contribution $\left(\zeta_{h}\right)$ to the friction agrees with the simulation results $\left(\zeta_{1}\right)$ better than the theoretical prediction $\left(\zeta_{t}\right)$ in the case without the randon shifting of the grid. The overall agreement between the simulation results and the theoretical predictions without and with the randon shifting of the grid is quite good.

\section{Conclusive Remarks}

We have provided hydrodynamic simulations of mesoscopic solvent by multi-particle collision algorithm in a real dimensional system without and with the random shifting of the grid. The system of the dimensionless units was successfully converted to a real dimensional system. The correct conversion was confirmed by jump rates of solvent particles at three different temperatures. Speed distributions of solvent particles obtained from the simulations agreed very well with the Maxwell-Boltzmann distributions. which means that the distribution relaxed rapidly to the equilibrium
Maxwell-Boltzmann form corresponding to the temperature $T$. Solvent viscosities calculated from the results of numerical simulations of the stress-stress auto-correlation functions were exactly the same as those obtained from the conversion of the units. This confirmed the correct conversion of units once again. The friction coefficient of a massive Brownian particle in a mesoscopic solvent was obtained as function of $\sigma$. The agreement between the simulation results and the theoretical predictions was quite good.

\section{References}

1. Frisch. U.: Hasslacher. B.: Pomeau. Y. Phus. Rev Lett. 1986. 56. 1505.

2. MoNamara G. R.: Zanetti. G. Phus. Rev Lett 1988, 61. 2332.

3. Pagonabarraga, I.: Hagen. M. H. J.: Frenkel. D. Ewophys. Lett. 1998. 42,377

4. Malevanets. A.: Kapral. R. J. Chent. Phos 1999. H0. 8605.

5. Malevanets. A.: Kapral. R. J. Chent. Phus 2000. 112. 7260.

6. Ihle. T.: Kroll. D. M. Phrs Rev E 2001. 63. 020201 .

7. Lamura A.: Gompper, G.: Ihle. T.: Kroll, D. M. Europhys. Lett. 2001. 56,768

8. Lamura A.: Gompper, G.: Ihle. T.: Kroll, D. M. Europhys, Lett. 2001. 56.319 .

9. Allahyarov. E.: Gompper. G. Phus Rev E 2002. 66.036702.

10. Lee. S. H.: Kapral. R. Phwsica $\$$ 2001. 298.56.

11. Ko. S. Y.: Lee. S. H. Bull Korean Chem. Soc. 2003. 24. 771.

12. Malevanets, A.: Yeomans, J. M. Ewophys. Lett. 2000. $52,231$.

13. Falck. E.: Lahtinen, J. M.: Vattulainen, I.: Ala-Nissila, T. Eu: Phus. J. E 2004. 13,67.

14. Hashimoto. Y: Chen. Y: Ohashi. H. Comp. Phns Comm 2000. 129.56.

15. Inoue. Y: Chen. Y:: Ohashi. H. Colloids and Surfaces A 2002. 201.297.

16. Sakai. T.; Chen. Y.: Ohashi. H. Plns. Rev E 2002, 65. 031503.

17. Tucei. K.: Kapral, R. J. Chem. Plins. 2004. 120,8262.

18. Lee. S. H.: Kapral. R. J. Chem. Phs 2004. 121. 1163.

19. Lee. S. H.: Kapral. R. In preparation.

20. Week. J. D.: Chandler. D.: Anderson. H. C. J. Chem. Phss 1971. 54. 5237.

21. Swope. W. C.: Andersen. H. C.: Berens. P. H.: Wilson. K. R. J. Chem. Phus. 1982, 76.673

22. Hynes. J. T.: Kapral. R.: Weinberg. M. J. Chent. Phis. 1970. 70. 1456. 